\title{
Sieve functions in arithmetic bands
}

\author{
Giovanni Coppola \& Maurizio Laporta
}

\begin{abstract}
An arithmetic function $f$ is a sieve function of range $Q$, if its Eratosthenes transform $g=f * \mu$ is supported in $[1, Q] \cap \mathbb{N}$, where $g(q) \ll_{\varepsilon} q^{\varepsilon}, \forall \varepsilon>0$. Here, we study the distribution of $f$ over the so-called short arithmetic bands $\bigcup_{1<a<H}\{n \in(N, 2 N]$ : $n \equiv a(\bmod q)\}$, with $H=o(N)$, and give applications to both the correlations and to the so-called weighted Selberg integrals of $f$, on which we have concentrated our recent research.
\end{abstract}

Keywords. mean squares, arithmetic progressions, short intervals.

2010 Mathematics Subject Classification. 11N37

\section{Contents}

1. Introduction and statement of the results $\ldots \ldots \ldots \ldots \ldots$

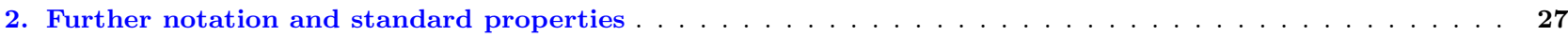

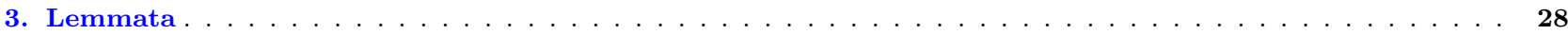

4. Proofs of Theorems 1.1, 1.9 and Corollary $1.7 \ldots \ldots \ldots \ldots$

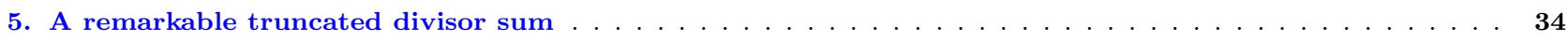

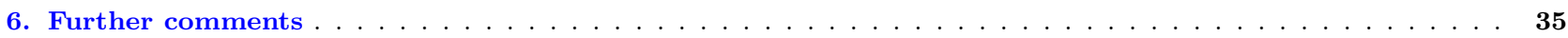

\section{Introduction and statement of the results}

If an arithmetic function $f: \mathbb{N} \rightarrow \mathbb{C}$ is the convolution product of $g$ and the constantly 1 function, i.e.

$$
f(n)=(g * \mathbf{1})(n)=\sum_{d \mid n} g(d),
$$

we say, with Wintner [Win43], that $g=f * \mu$ is the Eratosthenes transform of $f$ (where $\mu$ is the well-known Möbius function). We call $f$ a sieve function of range $Q$, if its Eratosthenes transform $g$ is essentially bounded, namely $g(d) \ll_{\varepsilon} d^{\varepsilon}, \forall \varepsilon>0$, and vanishes outside $[1, Q]$ for some $Q \in \mathbb{N}$, that is to say,

$$
f(n)=\sum_{\substack{d \mid n \\ d \leq Q}} g(d) .
$$

As usual, « is Vinogradov's notation, synonymous to Landau's $O$-notation. In particular, $\ll_{\varepsilon}$ means that the implicit constant might depend on an arbitrarily small and positive real number $\varepsilon$, which might change at each occurrence. We also write $g_{Q} \stackrel{\text { def }}{=} g \cdot \mathbf{1}_{[1, Q]}$ to mean that $g$ vanishes outside $[1, Q]$ (hereafter, $\mathbf{1}_{B}$ denotes the indicator function of the set $B \cap \mathbb{Z}$ ). Moreover, note that $f=g * \mathbf{1}$ is essentially bounded if and only if so is $g$.

Sieve functions are ubiquitous in analytic number theory. For example, the truncated divisor sum $\Lambda_{R}$, exploited by Goldston in [Gol92], is a linear combination of sieve functions of range $R$ (see Sect. 5.). Compare also [Cop10b] for more examples of sieve functions. However, the reader is cautioned that by a sieve function some authors simply mean any sieve-related function that often arises within the theory of sieve methods (see [DiaHal08]).

We thank episciences.org for providing open access hosting of the electronic journal Hardy-Ramanujan Journal 
The first author has intensively investigated symmetry properties of sieve functions in short intervals through the study of their correlations and the associated Selberg integrals ([Cop10a], [Cop10b] and [CopLap15]). Here we wish to relate such a study to the distribution of a sieve function in modular arithmetic short bands. More precisely, for given positive integers $q, N, H$ we search for non-trivial bounds on the total (balanced) value of $f$ in arithmetic bands modulo $q$ defined as

$$
T_{f}(q, N, H) \stackrel{\text { def }}{=} \sum_{a \leq H} \sum_{\substack{n \sim N \\ n \equiv a(\bmod q)}} f(n)-\frac{H}{q} \sum_{n \sim N} f(n),
$$

where $n \sim N$ means that $n \in(N, 2 N] \cap \mathbb{N}$ (hereafter, we omit $a \geq 1$ in sums like $\left.\sum_{a \leq H}\right)$. In particular, given any $N, H \in \mathbb{N}$, we prove that (see the remark after Theorem 1.1) for every real sieve function $f$ of range $Q \ll N$ and every $q \ll N$ one has

$$
T_{f}(q, N, H) \ll_{\varepsilon} N^{\varepsilon}(N / q+q+Q) .
$$

It transpires from our method that similar bounds can be immediately established for weighted versions of the above problem, namely

$$
T_{w, f}(q, N, H) \stackrel{\text { def }}{=} \sum_{0 \leq|a| \leq H} w(a) \sum_{\substack{n \sim N \\ n \equiv a(\bmod q)}} f(n)-\frac{1}{q} \sum_{0 \leq|h| \leq H} w(h) \sum_{n \sim N} f(n),
$$

whenever $w: \mathbb{R} \rightarrow \mathbb{R}$ is a piecewise-constant weight. Indeed, it is plain that $T_{f}(q, N, H)=T_{u, f}(q, N, H)$ involves the unit step weight

$$
u(h) \stackrel{\text { def }}{=}\left\{\begin{array}{l}
1 \text { if } h>0 \\
0 \text { otherwise. }
\end{array}\right.
$$

However, we give more general conditions on $w$ to treat $T_{w, f}(q, N, H)$. First, let us set

$$
\begin{gathered}
w_{H}(h) \stackrel{\text { def }}{=} w \cdot \mathbf{1}_{[-H, H]}(h)= \begin{cases}w(h) & \text { if } h \in[-H, H] \cap \mathbb{Z} \\
0 & \text { otherwise, }\end{cases} \\
\mathcal{L}_{\ell}^{1}\left(\widehat{w_{H}}\right) \stackrel{\text { def }}{=} \frac{1}{\ell} \sum_{\substack{j<\ell \\
(j, \ell)=1}}\left|\widehat{w_{H}}\left(\frac{j}{\ell}\right)\right|, \quad \text { where } \widehat{w_{H}}(\beta) \stackrel{\text { def }}{=} \sum_{0 \leq|h| \leq H} w(h) e(h \beta),
\end{gathered}
$$

(hereafter, $e(\alpha) \stackrel{\text { def }}{=} e^{2 \pi i \alpha} \forall \alpha \in \mathbb{R}$, and $(j, \ell) \stackrel{\text { def }}{=}$ g.c.d. $(j, \ell)$, as usual in number theory). Thus, we can write

$$
\sum_{a} w_{H}(a) \sum_{\substack{n \sim N \\ n \equiv a(\bmod q)}} f(n)=\frac{\widehat{w_{H}}(0)}{q} \sum_{n \sim N} f(n)+T_{w, f}(q, N, H)
$$

and state our first result.

Theorem 1.1. Let $q, N, H, Q$ be positive integers such that $q \ll N$ and $Q \ll N$, as $N \rightarrow \infty$. For every sieve function $f: \mathbb{N} \rightarrow \mathbb{R}$ of range $Q$ and every weight $w: \mathbb{R} \rightarrow \mathbb{R}$ one has

$$
T_{w, f}(q, N, H) \ll_{\varepsilon} N^{\varepsilon}\left(\frac{N}{q}+q+Q\right) \max _{\substack{\ell>1 \\ \ell \mid q}} \mathcal{L}_{\ell}^{1}\left(\widehat{w_{H}}\right) .
$$

Remark 1.2. By taking $w=u$ and recalling $\|r\| \stackrel{\text { def }}{=} \min _{n \in \mathbb{Z}}|r-n|, \forall r \in \mathbb{R}$, we have $\forall \ell>1$, (see [Da00], Ch.25),

$$
\mathcal{L}_{\ell}^{1}\left(\widehat{u_{H}}\right)=\frac{1}{\ell} \sum_{\substack{j<\ell \\(j, \ell)=1}}\left|\sum_{h \leq H} e\left(h \frac{j}{\ell}\right)\right| \ll \frac{1}{\ell} \sum_{\substack{j<\ell \\(j, \ell)=1}} \frac{1}{\|j / \ell\|} \ll \sum_{j \leq \ell / 2} \frac{1}{j} \ll \log \ell .
$$


Therefore, (1..1) follows immediately from Theorem 1.1.

Another remarkable instance concerns the correlation of $w_{H}$ given by

$$
W_{H}(a) \stackrel{\text { def }}{=} \sum_{\substack{h_{1} \\ h_{2}-h_{1}=a}} \sum_{h_{2}} w_{H}\left(h_{1}\right) w_{H}\left(h_{2}\right)=\sum_{\substack{0 \leq|h| \leq H \\ 0 \leq|h-a| \leq H}} w(h) w(h-a) .
$$

Note that $W_{H}$ vanishes outside $[-2 H, 2 H]$. Moreover, uniformly in $\beta \in[0,1]$, one has

$$
\begin{aligned}
\widehat{W_{H}}(\beta) & =\sum_{0 \leq|h| \leq 2 H} W_{H}(h) e(h \beta)=\sum_{h} \sum_{m-n=h} \sum_{H} w_{H}(m) w_{H}(n) e(h \beta) \\
& =\left|\sum_{r} w_{H}(r) e(r \beta)\right|^{2}=\left|\widehat{w_{H}}(\beta)\right|^{2} .
\end{aligned}
$$

Besides revealing that not all the weights are correlations of other weights, this yields

$$
\widehat{W_{H}}(0)=\widehat{w_{H}}(0)^{2} \ll H^{2}
$$

when $w_{H}$ is uniformly bounded as $H \rightarrow \infty$. Moreover, if $w_{H}$ also satisfies the inequality

$$
\mathcal{L}_{\ell}^{2}\left(\widehat{w_{H}}\right) \stackrel{\text { def }}{=} \frac{1}{\ell^{2}} \sum_{j<\ell}\left|\widehat{w_{H}}\left(\frac{j}{\ell}\right)\right|^{2} \ll \frac{H}{\ell}, \forall \ell \geq 1
$$

then

$$
\mathcal{L}_{\ell}^{1}\left(\widehat{W_{H}}\right)=\frac{1}{\ell} \sum_{\substack{j<\ell \\(j, \ell)=1}} \widehat{W_{H}}\left(\frac{j}{\ell}\right) \leq \ell \mathcal{L}_{\ell}^{2}\left(\widehat{w_{H}}\right) \ll H, \forall \ell \geq 1
$$

(Hereafter, $\diamond$ indicates the end of a remark).

According to [CopLap16], a uniformly bounded weight $w_{H}$ (as $H \rightarrow \infty$ ) is said to be good, if it satisfies (1..2). Thus, the following result is immediately established in a completely analogous way to the proof of Theorem 1.1.

Corollary 1.3. Let $q, N, H, Q$ be positive integers such that $q \ll N$ and $Q \ll N$, as $N \rightarrow \infty$. For every sieve function $f: \mathbb{N} \rightarrow \mathbb{R}$ of range $Q$ and every good weight $w: \mathbb{R} \rightarrow \mathbb{R}$ one has

$$
\sum_{a} W_{H}(a) \sum_{\substack{n \sim N \\ n \equiv a(\bmod q)}} f(n)=\frac{\widehat{W_{H}}(0)}{q} \sum_{n \sim N} f(n)+O_{\varepsilon}\left(N^{\varepsilon} H\left(\frac{N}{q}+q+Q\right)\right),
$$

where $W_{H}$ is the correlation of $w_{H}$.

Remark 1.4. Though analogous definitions can be easily formulated for a complex weight $w$ (with the only exception of $W_{H}$, whose definition has to be modified by taking the complex conjugate of $\left.w_{H}\left(h_{1}\right)\right)$, here we stick to real weights and real sieve functions for simplicity.

Remark 1.5. From [CopLap16] (see Propositions 2 and 3 there) it turns out that, beyond the unit step function $u$ defined above, other remarkable examples of good weights are the sign function and the Cesaro weight, respectively defined as

$$
\operatorname{sgn}(h) \stackrel{\text { def }}{=}\left\{\begin{array} { l l } 
{ 0 } & { \text { if } h = 0 } \\
{ h / | h | } & { \text { otherwise, } }
\end{array} \quad C _ { H } ( h ) \stackrel { \text { def } } { = } \left\{\begin{array}{ll}
1-|h| / H & \text { if }|h| \leq H \\
0 & \text { otherwise. }
\end{array}\right.\right.
$$


Since

$$
C_{H}(h)=\frac{1}{H} \sum_{t \leq H-|h|} 1=\frac{1}{H} \sum_{\substack{m, n \leq H \\ m-n=h}} \sum_{\substack{t \\ m-n}}
$$

then $H C_{H}$ is the correlation of $u_{H}$, and consequently $\widehat{C_{H}}(0)=\widehat{u_{H}}(0)^{2} / H=H$. We conclude that Corollary 1.3 is non-trivial for $w_{H}=u_{H}$, yielding

$$
\sum_{a} C_{H}(a) \sum_{\substack{n \sim N \\ n \equiv a(\bmod q)}} f(n)=\frac{H}{q} \sum_{n \sim N} f(n)+O_{\varepsilon}\left(N^{\varepsilon}\left(\frac{N}{q}+q+Q\right)\right) .
$$

Remark 1.6. The main terms in the formulæ furnished by Theorem 1.1 and Corollary 1.3 can be explicitly related to the Eratosthenes transform of $f=g_{Q} * \mathbf{1}$, with $Q \ll N$. Indeed,

$$
\begin{aligned}
\sum_{\substack{n \sim N \\
n \equiv a(\bmod q)}} f(n) & =\sum_{\substack{n \sim N \\
n \equiv a(\bmod q)}} \sum_{d \mid n} g_{Q}(d)=\sum_{d \leq Q} g(d) \sum_{\substack{n \sim N \\
n \equiv a(\bmod q) \\
n \equiv 0(\bmod d)}} 1 \\
& =\sum_{\substack{d \leq Q \\
(d \leq q) \mid a}} g(d) \sum_{\substack{n \sim N / d \\
n d \equiv a(\bmod q)}} 1=\sum_{\substack{d \leq Q \\
(d, q) \mid a}} g(d)\left(\frac{N}{d q}(d, q)+O(1)\right) \\
& =\frac{N}{q} \sum_{\substack{d \leq Q \\
(d, q) \mid a}} \frac{g(d)}{d}(d, q)+O_{\varepsilon}\left(Q^{1+\varepsilon}\right) .
\end{aligned}
$$

In particular, for the long intervals we get the formula

$$
\sum_{n \sim N} f(n)=R_{1}(f) N+O_{\varepsilon}\left(Q^{1+\varepsilon}\right)
$$

where the so-called first Ramanujan coefficient $R_{1}(f)$ is the mean value of $f$ (see Sect. 2.):

$$
\begin{aligned}
R_{1}(f) & \stackrel{\text { def }}{=} \sum_{d \leq Q} \frac{g(d)}{d} \\
& =\lim _{x \rightarrow \infty}\left(\sum_{d \leq Q} \frac{g(d)}{d}+\frac{1}{x} \sum_{d \leq Q} O(|g(d)|)\right)=\lim _{x \rightarrow \infty} \frac{1}{x} \sum_{n \leq x} f(n) .
\end{aligned}
$$

On the other side, by taking $F$ as the Dirichlet series generating $f$, one has

$$
\lim _{x \rightarrow \infty} \frac{1}{x} \sum_{n \leq x} f(n)=\operatorname{Res}_{s=1} F(s) \frac{x^{s-1}}{s} .
$$

Since $f=g_{Q} * \mathbf{1}$ is a sieve function, then $F$ can be expressed in terms of the Riemann zeta function $\zeta$ and the Dirichlet polynomial generating its Eratosthenes transform, namely

$$
F(s) \stackrel{\text { def }}{=} \sum_{n=1}^{\infty} \frac{f(n)}{n^{s}}=\zeta(s) \sum_{d \leq Q} \frac{g(d)}{d^{s}} .
$$

Note that the zeta function forces $F$ to have a simple pole at $s=1$, provided the $g$ series does not vanish at $s=1$. Thus, if $f=g_{Q} * \mathbf{1}$ is gauged by a weight $w$ in the short interval $[x-H, x+H]$ 
(i.e. $H=o(N)$, as $N \rightarrow \infty)$, then it is natural to take the expected mean value of $w_{H}(n-x) f(n)$ for $N<x \leq 2 N$ to be (compare [CopLap14])

$$
\left.\widehat{w_{H}}(0) R_{1}(f)=\sum_{a} w_{H}(a) \sum_{d \leq Q} \frac{g(d)}{d} \quad \text { (that is independent of } x\right) .
$$

Indeed, a basic tool for the study of the distribution of the sieve function $f$ in short intervals is its weighted Selberg integral

$$
J_{w, f}(N, H) \stackrel{\text { def }}{=} \sum_{x \sim N}\left|\sum_{n} w_{H}(n-x) f(n)-\widehat{w_{H}}(0) R_{1}(f)\right|^{2},
$$

whose non-trivial bounds might lead to results on the distribution of $f$ in almost all short intervals $[x-H, x+H]$, i.e. with $o(N)$ possible exceptions $x \in(N, 2 N] \cap \mathbb{N}$. Observe that the trivial bound for $J_{w, f}(N, H)$ is $N^{1+\varepsilon} H^{2}$, because $f$ is essentially bounded. In [CopLap14] and [CopLap16] we have investigated and exploited the link between $J_{w, f}(N, H)$ and the correlation

$$
\mathcal{C}_{f}(a) \stackrel{\text { def }}{=} \sum_{n \sim N} f(n) f(n-a)
$$

in order to get non-trivial bounds under suitable conditions on $f$ and a good weight $w$.

As a consequence of Theorem 1.1, we obtain a further result on such a link with a slight generalization. Let us define the correlation of real arithmetic functions $f_{1}$ and $f_{2}$ as

$$
\mathcal{C}_{f_{1}, f_{2}}(a) \stackrel{\text { def }}{=} \sum_{n \sim N} f_{1}(n) f_{2}(n-a) .
$$

In such a context, we might refer to $\mathcal{C}_{f}=\mathcal{C}_{f, f}$ as the autocorrelation of $f$. Since the shift a is confined to $a \ll H$, the conditions $n \sim N$ and $H=o(N)$ clearly yield $\max (n, n-a) \leq 2 N+|a| \leq 3 N$. Moreover, if $f_{1}$ and $f_{2}$ are essentially bounded, then trivially $\mathcal{C}_{f_{1}, f_{2}}(0) \ll N^{1+\varepsilon}$, and for any $a \ll H$ one has

$$
\mathcal{C}_{f_{1}, f_{2}}(a)=\sum_{\substack{n_{1} \sim N \\ n_{2}-n_{1}=a}} \sum_{\substack{n_{2} \sim N \\ n_{1}}} f_{1}\left(n_{1}\right) f_{2}\left(n_{2}\right)+O_{\varepsilon}\left(N^{\varepsilon} H\right)
$$

(to be compared to the previous definition of the correlation of a weight).

Correspondingly, the mixed weighted Selberg integral associated to the pair $\left(f_{1}, f_{2}\right)$ is (compare [Cop14])

$$
J_{w,\left(f_{1}, f_{2}\right)}(N, H) \stackrel{\text { def }}{=} \sum_{x \sim N} \prod_{j=1,2}\left(\sum_{n} w_{H}(n-x) f_{j}(n)-\widehat{w_{H}}(0) R_{1}\left(f_{j}\right)\right) .
$$

By assuming that $f_{2}=g_{2} * \mathbf{1}$ is a sieve function of range $Q_{2}$ it is readily seen that (see also the proof of Lemma 3.3 below)

$$
\mathcal{C}_{f_{1}, f_{2}}(a)=\sum_{q \leq Q_{2}} g_{2}(q) \sum_{\substack{n \sim N \\ n \equiv a(q)}} f_{1}(n) .
$$

Moreover, Lemma 3.3 also shows that if $f_{1}$ and $f_{2}$ are sieve functions, then $J_{w,\left(f_{1}, f_{2}\right)}(N, H)$ can be expressed in terms of arithmetic bands of $f_{1}$ or $f_{2}$. Such favorable circumstances allow us to apply Theorem 1.1 in order to obtain both a so-called first generation formula (consistently with the terminology of [CopLap14]) for the correlation of the sieve functions $f_{1}$ and $f_{2}$ (more precisely, here we use (1..1) that is a consequence of Theorem 1.1 as it is showed in Remark 1.2) and an estimate of $J_{w,\left(f_{1}, f_{2}\right)}(N, H)$ once $f_{1}$ and $f_{2}$ are gauged by a good weight $w$. 
Corollary 1.7. Let $N, H, Q_{1}, Q_{2}$ be positive integers with $Q_{1} \leq Q_{2} \ll N$, as $N \rightarrow \infty$. For any real and essentially bounded arithmetic functions $g_{1}$ and $g_{2}$ supported in $\left[1, Q_{1}\right]$ and $\left[1, Q_{2}\right]$, respectively, one has

$$
\sum_{a \leq H} \mathcal{C}_{f_{1}, f_{2}}(a)=R_{1}\left(f_{1}\right) R_{1}\left(f_{2}\right) N H+O_{\varepsilon}\left(N^{\varepsilon}\left(N+Q_{2}^{2}+Q_{1} H\right)\right),
$$

where $f_{j}=g_{j} * \mathbf{1}$ for $j=1,2$. Furthermore, if $H=o(N)$, as $N \rightarrow \infty$, and $w: \mathbb{R} \rightarrow \mathbb{R}$ is a good weight, then

$$
J_{w,\left(f_{1}, f_{2}\right)}(N, H) \ll_{\varepsilon} N^{\varepsilon}\left(N H+Q_{2} H^{2}+Q_{2}^{2} H+H^{3}\right) .
$$

Remark 1.8. For every real sieve function $f$ of range $Q \ll N$, this corollary gives

$$
\begin{gathered}
\sum_{a \leq H} \mathcal{e}_{f}(a)=R_{1}^{2}(f) N H+O_{\varepsilon}\left(N^{\varepsilon}\left(N+Q^{2}+Q H\right)\right), \\
J_{w, f}(N, H) \ll_{\varepsilon} N^{\varepsilon}\left(N H+Q H^{2}+Q^{2} H+H^{3}\right) .
\end{gathered}
$$

We stress that such a bound for the weighted Selberg integral has been already established in Theorem 3 of [CopLap16]. In Sect. 4. we propose a much simpler proof through the new approach of the arithmetic bands formulæ provided by Theorem 1.1.

Furthermore, from such an approach we find an important relation between weighted Selberg integrals and the total (weighted) content of a sieve function $f$ of range $Q \ll N$, namely (see Lemma 3.3 and the proof of Corollary 1.7)

$$
J_{w, f}(N, H) \ll_{\varepsilon} N^{\varepsilon} \sum_{q \leq Q}\left|T_{W, f}(q, N, H)\right|+N^{\varepsilon} H^{2}(Q+H),
$$

where for the correlation of $w_{H}$ we set

$$
T_{W, f}(q, N, H) \stackrel{\text { def }}{=} \sum_{a} W_{H}(a) \sum_{\substack{n \sim N \\ n \equiv a(\bmod q)}} f(n)-\frac{\widehat{W_{H}}(0)}{q} \sum_{n \sim N} f(n) .
$$

Beyond Corollary 1.3, more generally, given an essentially bounded $f$, a non-trivial bound like

$$
\sum_{q \leq Q}\left|T_{W, f}(q, N, H)\right| \ll N^{1-\delta} H^{2}, \text { for some real } \delta>0,
$$

might yield a non-trivial bound of the same type for $J_{w, f}(N, H)$ (but not necessarily with the same gain $N^{\delta}$ ) by means of (1..4). Analogous considerations hold for mixed weighted Selberg integrals. Rather surprisingly, in spite of the fact that the presence of absolute values in the total content seems to prevent it from further possible cancellation, the next theorem makes it clear that there are nontrivial bounds for (weighted) Selberg integrals, involving a sieve function $f$ of range $Q \ll N^{1-\delta}$ for some $\delta>0$, if and only if there are non-trivial results on the distribution of $f$ in short arithmetic bands.

Theorem 1.9. Let $f: \mathbb{N} \rightarrow \mathbb{R}$ be a sieve function of range $Q \ll N^{1-\delta}$, for some $\delta>0$, and let $w: \mathbb{R} \rightarrow \mathbb{R}$ be such that $w_{H}$ is uniformly bounded for any $H \ll N^{1-\delta}$, as $N \rightarrow \infty$.

I) The following three assertions are equivalent:

i) a non-trivial bound holds for $\sum_{q \leq Q}\left|T_{W, f}(q, N, H)\right|$ 
ii) a non-trivial bound holds for $J_{w, f}(N, H)$

iii) a non-trivial bound holds for $J_{w,\left(f, f_{1}\right)}(N, H)$, where $f_{1}$ is any sieve function of range $Q$.

II) If $N^{\delta / 2} \ll H \ll N^{1-\delta}$, as $N \rightarrow \infty$, then the following assertions are equivalent:

iv) a non-trivial bound holds for $\sum_{q \leq Q}\left|T_{f}(q, N, H)\right|$

v) a non-trivial bound holds for the Selberg integral

$$
J_{f}(N, H) \stackrel{\text { def }}{=} \sum_{x \sim N}\left|\sum_{x<n \leq x+H} f(n)-R_{1}(f) H\right|^{2} .
$$

Note that in $i v$ ) a non-trivial bound is meant to be of the type $N^{1-\delta} H$ for some $\delta>0$.

After a short section on some further notation and basic formulæ, in Sect. 3. we give the necessary lemmata for our theorems and for Corollary 1.7, whose proofs constitute the fourth section, whereas we omit the proof of Corollary 1.3, it being completely analogous to the proof of Theorem 1.1. In Sect. 4. we specialize the results of the present article to the aforementioned function $\Lambda_{R}$. The last section is devoted to a comparison between classical results in arithmetic progressions and ours in arithmetic bands.

\section{Further notation and standard properties}

As already mentioned, we omit $a \geq 1$ in sums like $\sum_{a \leq X}$. For the same sake of brevity, at times we write $n \equiv a(q)$ in place of $n \equiv a(\bmod q)$. Thus, the well-known orthogonality of additive characters,

can be written as

$$
e_{q}(r) \stackrel{\text { def }}{=} e(r / q)=e^{2 \pi i r / q},(q \in \mathbb{N}, r \in \mathbb{Z}),
$$

$$
\frac{1}{q} \sum_{j(q)} e_{q}(j(n-m))=\frac{1}{q} \sum_{j \leq q} e_{q}(j(n-m))=\left\{\begin{array}{l}
1 \text { if } n \equiv m(\bmod q) \\
0 \text { otherwise }
\end{array},\right.
$$

since the sum is over a complete set of residue classes $j(\bmod q)$.

We write $\sum_{j(q)}^{*}$ to mean that the sum is over a complete set of reduced residue classes $(\bmod q)$, i.e. the set $\mathbb{Z}_{q}^{*}$ of $1 \leq j \leq q$ such that $(j, q)=1$. In particular, the Ramanujan sum is written as

$$
c_{q}(n) \stackrel{\text { def }}{=} \sum_{j(q)}^{*} e_{q}(j n)
$$

Without further references, we will appeal to the well-known inequality (see [Da00], Ch.25)

$$
\sum_{V_{1}<v \leq V_{2}} e(v \alpha) \ll \min \left(V_{2}-V_{1}, \frac{1}{\|\alpha\|}\right) .
$$

Recalling that $\mathbf{1}(n) \stackrel{\text { def }}{=} 1, \forall n \in \mathbb{N}$, we set

$$
\mathbf{1}_{d \mid n} \stackrel{\text { def }}{=}\left\{\begin{array}{l}
1 \text { if } d \mid n \\
0 \text { otherwise. }
\end{array}\right.
$$


Consequently, the aforementioned orthogonality of characters becomes

$$
\mathbf{1}_{d \mid n}=\frac{1}{d} \sum_{j^{\prime}(d)} e_{d}\left(j^{\prime} n\right)=\frac{1}{d} \sum_{\ell \mid d} \sum_{\substack{j^{\prime}(d) \\\left(j^{\prime}, d\right)=d / \ell}} e_{d}\left(j^{\prime} n\right)=\frac{1}{d} \sum_{\ell \mid d} c_{\ell}(n) .
$$

Therefore, one has the following Ramanujan expansion of a sieve function $f=g_{Q} * \mathbf{1}$ :

$$
\begin{aligned}
f(n) & =\sum_{d \mid n} g_{Q}(d)=\sum_{d \leq Q} g(d) \mathbf{1}_{d \mid n}=\sum_{d \leq Q} \frac{g(d)}{d} \sum_{\ell \mid d} c_{\ell}(n) \\
& =\sum_{\ell \leq Q} \sum_{\substack{d \leq Q \\
d \equiv 0(\ell)}} \frac{g(d)}{d} c_{\ell}(n)=\sum_{\ell \leq Q} R_{\ell}(f) c_{\ell}(n),
\end{aligned}
$$

where we have introduced the so-called $\ell$-th Ramanujan coefficient of $f$, i.e.

$$
R_{\ell}(f) \stackrel{\text { def }}{=} \sum_{d \equiv 0(\ell)} \frac{g_{Q}(d)}{d} .
$$

The hypothesis that $g$ is essentially bounded yields the bound

$$
R_{\ell}(f) \ll \frac{1}{\ell} \sum_{m \leq \frac{Q}{\ell}} \frac{|g(\ell m)|}{m} \ll_{\varepsilon} \frac{Q^{\varepsilon}}{\ell} \sum_{m \leq \frac{Q}{\ell}} \frac{1}{m} \ll_{\varepsilon} \frac{Q^{\varepsilon}}{\ell} .
$$

We refer the reader to [SchSpi94] for more extensive accounts on the theory of the Ramanujan expansions.

\section{Lemmata}

Here we state and prove two lemmas that are interesting in their own right. The first lemma is required to prove Theorem 1.1, while the second one is invoked within the proofs of Corollary 1.7 and Theorem 1.9. To this end, analogously to the exponential sums for the weights already introduced in Sect. 1., we set

$$
\widehat{f}(\alpha) \stackrel{\text { def }}{=} \sum_{n \sim N} f(n) e(n \alpha) \quad(\alpha \in \mathbb{R}) .
$$

Notice that now we can write

$$
\begin{aligned}
T_{w, f}(q, N, H) & =\sum_{a} w_{H}(a) \sum_{\substack{n \sim N \\
n \equiv a(q)}} f(n)-\widehat{w_{H}}(0) \frac{\widehat{f}(0)}{q}, \\
T_{W, f}(q, N, H) & =\sum_{a} W_{H}(a) \sum_{\substack{n \sim N \\
n \equiv a(q)}} f(n)-\widehat{W_{H}}(0) \frac{\widehat{f}(0)}{q},
\end{aligned}
$$

while the formula (1..3) becomes

$$
\widehat{f}(0)=R_{1}(f) N+O_{\varepsilon}\left(Q^{1+\varepsilon}\right) .
$$

The first lemma gives a similar relation between the $\ell$-th Ramanujan coefficient of $f$ and $\widehat{f}(\alpha)$, when $\alpha=j / \ell$ is any non-integer rational with $(j, \ell)=1$. Note that such a formula is not a straightforward consequence of Wintner's criterion (see VIII.2 of [SchSpi94]). 
Lemma 3.1. Let $f$ be a sieve function of range $Q \ll N$, with $Q, N \in \mathbb{N}$. Then

$$
\widehat{f}(j / \ell)=R_{\ell}(f) N+O_{\varepsilon}\left((\ell Q)^{\varepsilon}(Q+\ell)\right), \quad \forall \ell>1, \forall j \in \mathbb{Z}_{\ell}^{*}
$$

Proof. By hypothesis $f=g_{Q} * \mathbf{1}$ with an essentially bounded $g$. Hence

$$
\begin{aligned}
\widehat{f}(j / \ell) & =\sum_{d} g_{Q}(d) \sum_{v \sim \frac{N}{d}} e_{\ell}(j d v) \\
& =\sum_{d \equiv 0(\ell)} g_{Q}(d)\left(\frac{N}{d}+O(1)\right)+O\left(\sum_{d \neq 0(\ell)} \frac{\left|g_{Q}(d)\right|}{\|j d / \ell\|}\right) .
\end{aligned}
$$

Since

$$
\sum_{d \equiv 0(\ell)} g_{Q}(d)\left(\frac{N}{d}+O(1)\right)=R_{\ell}(f) N+O_{\varepsilon}\left(Q^{\varepsilon}\left(\frac{Q}{\ell}+1\right)\right),
$$

then the lemma is proved whenever we show that

$$
\sum_{\substack{d \leq Q \\ d \neq 0(\ell)}} \frac{1}{\|j d / \ell\|} \ll_{\varepsilon} \ell^{\varepsilon}(Q+\ell) .
$$

To this end, it suffices to observe that

$$
\sum_{\substack{d \leq Q \\ d \neq 0(\ell)}} \frac{1}{\|j d / \ell\|} \leq \sum_{0<|r| \leq \ell / 2} \sum_{\substack{d \leq Q \\ j d \equiv r(\ell)}} \frac{1}{|r / \ell|} \ll \ell \sum_{r \leq \ell / 2} \frac{1}{r}\left(\frac{Q}{\ell}+1\right) .
$$

The proof is completed.

Remark 3.2. Note that the formula of the above lemma is non-trivial when $\ell, Q \ll N^{1-\delta}$, for some $\delta>0$. Moreover, it is easy to see that it holds uniformly with respect to $j \in \mathbb{Z}_{\ell}^{*}$.

Let us turn our attention to the next lemma. As already mentioned in Sect. 1., by means of an elementary dispersion method, in [CopLap14], Lemma 7, we established a link between weighted Selberg integrals and autocorrelations of an arithmetic function $f$ gauged by a weight $w$ such that $w_{H}$ is bounded, as $H \rightarrow \infty$. Under the further hypothesis that the sieve function $f$ and the weight $w$ are real, the formula of the aforementioned lemma becomes

$$
\begin{aligned}
J_{w, f}(N, H)= & \sum_{0 \leq|a| \ll H} W_{H}(a) \mathcal{C}_{f}(a)+\sum_{x \sim N}\left|\widehat{w_{H}}(0) R_{1}(f)\right|^{2} \\
& -2 \widehat{w_{H}}(0) R_{1}(f) \sum_{n \leq 3 N} f(n) \sum_{x \sim N} w_{H}(n-x)+O_{\varepsilon}\left(H^{3} N^{\varepsilon}\right) .
\end{aligned}
$$

Similarly, for the mixed weighted Selberg integral of sieve functions $f_{1}, f_{2}$ we have

$$
\begin{aligned}
J_{w,\left(f_{1}, f_{2}\right)}(N, H)= & \sum_{a} W_{H}(a) \mathcal{C}_{f_{1}, f_{2}}(a)-\widehat{W_{H}}(0) R_{1}\left(f_{1}\right) R_{1}\left(f_{2}\right) N \\
& -\widehat{w_{H}}(0)\left(R_{1}\left(f_{1}\right) \sum_{x \sim N} \Delta_{2}(x)+R_{1}\left(f_{2}\right) \sum_{x \sim N} \Delta_{1}(x)\right) \\
& +O_{\varepsilon}\left(H^{3} N^{\varepsilon}\right),
\end{aligned}
$$

where we set $\Delta_{j}(x) \stackrel{\text { def }}{=} \sum_{n} w_{H}(n-x) f_{j}(n)-\widehat{w_{H}}(0) R_{1}\left(f_{j}\right)$.

By using such a formula we prove the next lemma, where $J_{w,\left(f_{1}, f_{2}\right)}(N, H)$ is expressed in terms of arithmetic bands of $f_{1}$ or $f_{2}$. 
Lemma 3.3. Let $g_{1}$ and $g_{2}$ be real and essentially bounded arithmetic functions supported in $\left[1, Q_{1}\right]$ and $\left[1, Q_{2}\right]$, respectively, with $Q_{1}, Q_{2} \in \mathbb{N}$ such that $Q_{1} \leq Q_{2} \ll N$, as $N \rightarrow \infty$. If $w: \mathbb{R} \rightarrow \mathbb{R}$ is such that $w_{H}$ is uniformly bounded, as $H \rightarrow \infty$, then one has

$$
\begin{aligned}
J_{w,\left(f_{1}, f_{2}\right)}(N, H) & =\sum_{q \leq Q_{1}} g_{1}(q) T_{W, f_{2}}(q, N, H)+O_{\varepsilon}\left(N^{\varepsilon} H^{2}\left(Q_{2}+H\right)\right) \\
& =\sum_{q \leq Q_{2}} g_{2}(q) T_{W, f_{1}}(q, N, H)+O_{\varepsilon}\left(N^{\varepsilon} H^{2}\left(Q_{2}+H\right)\right),
\end{aligned}
$$

where we set $f_{j}=g_{j} * \mathbf{1}$, and $W_{H}$ is the correlation of $w_{H}$.

Proof. First, let us write

$$
\begin{aligned}
\sum_{x \sim N} \sum_{n} w_{H}(n-x) f_{j}(n) & =\sum_{n \sim N} f_{j}(n) \sum_{n-H \leq x \leq n+H} w(n-x)+O_{\varepsilon}\left(N^{\varepsilon} H^{2}\right) \\
& =\widehat{w_{H}}(0) \sum_{n \sim N} f_{j}(n)+O_{\varepsilon}\left(N^{\varepsilon} H^{2}\right) .
\end{aligned}
$$

Then, by arguing as in (1..3) and recalling that $R_{1}\left(f_{j}\right) \ll_{\varepsilon} Q_{j}^{\varepsilon}$, we get

$$
\begin{aligned}
\sum_{x \sim N} \Delta_{j}(x) & =\widehat{w_{H}}(0)\left(\sum_{n \sim N} f_{j}(n)-R_{1}\left(f_{j}\right) N\right)+O_{\varepsilon}\left(N^{\varepsilon} H^{2}\right) \\
& \ll_{\varepsilon} N^{\varepsilon} H\left(Q_{j}+H\right) .
\end{aligned}
$$

Since $W_{H}$ is even and $Q_{1} \leq Q_{2} \ll N$, the above formula (3..1) yields

$$
\begin{aligned}
J_{w,\left(f_{1}, f_{2}\right)}(N, H)= & \sum_{a} W_{H}(a) \mathcal{C}_{f_{1}, f_{2}}(a)-\widehat{W_{H}}(0) R_{1}\left(f_{1}\right) R_{1}\left(f_{2}\right) N \\
& +O_{\varepsilon}\left(N^{\varepsilon} H^{2}\left(Q_{2}+H\right)\right) \\
= & \sum_{a} W_{H}(a) \mathcal{C}_{f_{2}, f_{1}}(a)-\widehat{W_{H}}(0) R_{1}\left(f_{1}\right) R_{1}\left(f_{2}\right) N \\
& +O_{\varepsilon}\left(N^{\varepsilon} H^{2}\left(Q_{2}+H\right)\right) .
\end{aligned}
$$

Thus, we can stick to the first equality, apply (1..3) to $f_{1}$ and write

$$
\begin{aligned}
& \sum_{a} W_{H}(a) \mathcal{C}_{f_{1}, f_{2}}(a)-\widehat{W_{H}}(0) R_{1}\left(f_{1}\right) R_{1}\left(f_{2}\right) N \\
& =\sum_{a} W_{H}(a) \sum_{n \sim N} f_{1}(n) \sum_{\substack{q \mid n-a \\
q \leq Q_{2}}} g_{2}(q) \\
& -\widehat{W_{H}}(0) \sum_{n \sim N} f_{1}(n) \sum_{q \leq Q_{2}} \frac{g_{2}(q)}{q}+O_{\varepsilon}\left(Q_{2}^{1+\varepsilon} H^{2}\right) \\
& =\sum_{q \leq Q_{2}} g_{2}(q)\left(\sum_{a} W_{H}(a) \sum_{\substack{n \sim N \\
n \equiv a(q)}} f_{1}(n)-\widehat{W_{H}}(0) \frac{\widehat{f}_{1}(0)}{q}\right) \\
& +O_{\varepsilon}\left(Q_{2}^{1+\varepsilon} H^{2}\right) \text {. }
\end{aligned}
$$

The lemma is completely proved. 


\section{Proofs of Theorems 1.1, 1.9 and Corollary 1.7}

Proof of Theorem 1.1. By the orthogonality of additive characters we get

$$
\begin{aligned}
T_{w, f}(q, N, H) & =\frac{1}{q} \sum_{a} w_{H}(a) \sum_{n \sim N} f(n) \sum_{j^{\prime} \leq q} e_{q}\left(j^{\prime}(a-n)\right)-\frac{\widehat{w_{H}}(0)}{q} \widehat{f}(0) \\
& =\frac{1}{q} \sum_{j^{\prime}<q} \sum_{a} w_{H}(a) e_{q}\left(j^{\prime} a\right) \widehat{f}\left(-j^{\prime} / q\right) \\
& =\frac{1}{q} \sum_{\ell>1} \sum_{j(\ell)}^{*} \widehat{f}(-j / \ell) \widehat{w_{H}}(j / \ell)
\end{aligned}
$$

where we have set $\ell=q /\left(j^{\prime}, q\right)$. By applying Lemma 3.1 and $(2 . .1)$ we see that

$$
\begin{aligned}
T_{w, f}(q, N, H) & \ll_{\varepsilon} \frac{1}{q} \sum_{\substack{\ell>1 \\
\ell \mid q}}\left(\left|R_{\ell}(f)\right| N+(\ell Q)^{\varepsilon}(Q+\ell)\right) \sum_{j(\ell)}^{*}\left|{\widehat{w_{H}}}\left(\frac{j}{\ell}\right)\right| \\
& \ll_{\varepsilon} \frac{Q^{\varepsilon}}{q} \sum_{\substack{\ell>1 \\
\ell \mid q}}\left(\frac{N}{\ell}+Q \ell^{\varepsilon}+\ell^{1+\varepsilon}\right) \ell \mathcal{L}_{\ell}^{1}\left(\widehat{w_{H}}\right) \\
& \ll_{\varepsilon} N^{\varepsilon}\left(\frac{N}{q}+Q+q\right) \max _{\substack{\ell>1 \\
\ell \mid q}} \mathcal{L}_{\ell}^{1}\left(\widehat{w_{H}}\right) .
\end{aligned}
$$

The theorem is completely proved.

Proof of Corollary 1.7. As already noticed in the proof of Lemma 3.3, we can write

$$
\begin{aligned}
\mathcal{C}_{f_{1}, f_{2}}(a) & =\sum_{n \sim N} f_{1}(n) f_{2}(n-a)=\sum_{n \sim N} f_{1}(n) \sum_{\substack{q \mid n-a \\
q \leq Q_{2}}} g_{2}(q) \\
& =\sum_{q \leq Q_{2}} g_{2}(q) \sum_{\substack{n \sim N \\
n \equiv a(q)}} f_{1}(n) .
\end{aligned}
$$

Thus, the formula (1..3) and Theorem 1.1 (more precisely, we apply (1..1) here) yield

$$
\begin{aligned}
\sum_{a \leq H} \mathcal{e}_{f_{1}, f_{2}}(a)= & \sum_{q \leq Q_{2}} g_{2}(q)\left(\frac{H}{q} \widehat{f}_{1}(0)+T_{f_{1}}(q, N, H)\right) \\
= & H\left(\sum_{q \leq Q_{2}} \frac{g_{2}(q)}{q}\right)\left(R_{1}\left(f_{1}\right) N+O_{\varepsilon}\left(Q_{1}^{1+\varepsilon}\right)\right) \\
& +O_{\varepsilon}\left(N^{\varepsilon} \sum_{q \leq Q_{2}}\left(\frac{N}{q}+q+Q_{1}\right)\right) \\
= & R_{1}\left(f_{1}\right) R_{1}\left(f_{2}\right) N H+O_{\varepsilon}\left(N^{\varepsilon}\left(N+Q_{2}^{2}+Q_{1} H\right)\right),
\end{aligned}
$$

that is the first formula of Corollary 1.7. In order to prove the stated inequality for the mixed weighted Selberg integral, it is enough to observe that Lemma 3.3 and the hypothesis $Q_{1} \leq Q_{2} \ll N$ imply

$$
\begin{aligned}
J_{w,\left(f_{1}, f_{2}\right)}(N, H) & =\sum_{q \leq Q_{2}} g_{2}(q) T_{W, f_{1}}(q, N, H)+O_{\varepsilon}\left(N^{\varepsilon} H^{2}\left(Q_{2}+H\right)\right) \\
& \ll_{\varepsilon} \quad N^{\varepsilon} \sum_{q \leq Q_{2}}\left|T_{W, f_{1}}(q, N, H)\right|+N^{\varepsilon} H^{2}\left(Q_{2}+H\right) .
\end{aligned}
$$


Hence the conclusion follows immediately from Corollary 1.3.

Before going to the proof of Theorem 1.9, let us remark explicitly that (1..4) is plainly a particular case of the latter inequality. Moreover, it transpires from the previous proof that, given any real and essentially bounded arithmetic function $g$ supported in $[1, Q]$, with $Q \ll N$, for $f=g * \mathbf{1}$ one has

$$
\sum_{a \leq H} \mathcal{e}_{f}(N)=R_{1}(f)^{2} N H+\sum_{q \leq Q} g(q) T_{f}(q, N, H)+O_{\varepsilon}\left(N^{\varepsilon} Q H\right)
$$

Proof of Theorem 1.9. For simplicity and without loss of generality, let us assume that, whatever the choice of an assertion among $i$ )-v) as hypothesis, the gain of the non-trivial bound is always $N^{\delta}$.

Part I. $i) \Longrightarrow i i)$ : as we said, let us suppose that

$$
\sum_{q \leq Q}\left|T_{W, f}(q, N, H)\right| \ll N^{1-\delta} H^{2} .
$$

Thus, $i i)$ follows immediately from (1..4), where $H^{2}(Q+H) \ll N^{1-\delta} H^{2}$ because of the hypotheses $H, Q \ll N^{1-\delta}$.

ii) $\Longrightarrow$ iii): since we assume that $J_{w, f}(N, H) \ll N^{1-\delta} H^{2}$, then by the Cauchy inequality and the trivial bound for $J_{w, f_{1}}(N, H)$ we get

$$
\begin{aligned}
J_{w,\left(f, f_{1}\right)}(N, H) & \leq \sqrt{J_{w, f}(N, H)} \sqrt{J_{w, f_{1}}(N, H)} \\
& \ll_{\varepsilon} N^{\varepsilon} \sqrt{N^{1-\delta} H^{2}} \sqrt{N H^{2}} \ll N^{1-\delta / 3} H^{2} .
\end{aligned}
$$

iii) $\Longrightarrow i$ ): after setting

$$
s_{W, f}(q) \stackrel{\text { def }}{=} \begin{cases}\operatorname{sgn}\left(T_{W, f}(q, N, H)\right) & \text { if } 1 \leq q \leq Q \\ 0 & \text { otherwise }\end{cases}
$$

it is readily seen that $f_{1}=s_{W, f} * \mathbf{1}$ is a sieve function of range $Q$. Thus, we can write

$$
\sum_{q \leq Q}\left|T_{W, f}(q, N, H)\right|=\sum_{q} s_{W, f}(q) T_{W, f}(q, N, H) .
$$

Now, by taking $g_{1}=s_{W, f}$ and $f_{2}=f$ in Lemma 3.3 we see that

$$
\sum_{q \leq Q}\left|T_{W, f}(q, N, H)\right|=J_{w,\left(f, f_{1}\right)}(N, H)+O_{\varepsilon}\left(N^{\varepsilon} H^{2}(Q+H)\right),
$$

where again $H^{2}(Q+H)$ is non-trivial. The first part of the theorem is completely proved.

Part II. $i v) \Longrightarrow v$ ): since $Q \ll N^{1-\delta}$ and we assume that

$$
\sum_{q \leq Q}\left|T_{f}(q, N, H)\right| \ll N^{1-\delta} H,
$$

then it is easily seen that the formula (4..1) yields

$$
\sum_{a \leq t} \mathcal{C}_{f}(a)=R_{1}(f)^{2} N[t]+O_{\varepsilon}\left(N^{1-\delta+\varepsilon} t\right) \quad \text { for all } 1 \leq t \leq H
$$


where $[t]$ is the integer part of $t$. Thus, by partial summation we can write

$$
\begin{aligned}
\sum_{1 \leq a \leq H}(H-a) \mathfrak{e}_{f}(a) & =\int_{1}^{H} \sum_{a \leq t} \mathfrak{e}_{f}(a) d t \\
& =\int_{1}^{H}\left(R_{1}(f)^{2} N[t]+O_{\varepsilon}\left(N^{1-\delta+\varepsilon} t\right)\right) d t \\
& =\frac{R_{1}(f)^{2}}{2} N H^{2}+O_{\varepsilon}\left(N^{1+\varepsilon} H\right)+O_{\varepsilon}\left(N^{1-\delta+\varepsilon} H^{2}\right) .
\end{aligned}
$$

Now, since $\mathcal{C}_{f}(0) \ll_{\varepsilon} N^{1+\varepsilon}$, and for $1 \leq a \leq H$ one has

$$
\begin{aligned}
\mathcal{C}_{f}(-a) & =\sum_{n \sim N} f(n) f(n+a)=\sum_{N+a<m \leq 2 N+a} f(m-a) f(m) \\
& =\mathcal{C}_{f}(a)+O_{\varepsilon}\left(N^{\varepsilon} H\right),
\end{aligned}
$$

then

$$
\sum_{0 \leq|a| \leq H}(H-|a|) \mathcal{C}_{f}(a)=R_{1}(f)^{2} N H^{2}+O\left(N^{1-\delta / 3} H^{2}\right) .
$$

By using this formula in (3..1), where we take

$$
W_{H}(a)=H C_{H}(a)=\max (H-|a|, 0)
$$

(see Remark 1.5), we immediately obtain $J_{f}(N, H) \ll N^{1-\delta / 3} H^{2}$.

$v) \Longrightarrow i v)$ : we suppose that $J_{f}(N, H) \ll N^{1-\delta} H^{2}$ and set

$$
s_{f}(q) \stackrel{\text { def }}{=}\left\{\begin{array}{ll}
\operatorname{sgn}\left(T_{f}(q, N, H)\right) & \text { if } 1 \leq q \leq Q, \\
0 & \text { otherwise }
\end{array} \quad f_{1} \stackrel{\text { def }}{=} s_{f} * \mathbf{1} .\right.
$$

Thus, we can write

$$
\begin{aligned}
\sum_{q \leq Q}\left|T_{f}(q, N, H)\right|= & \sum_{q} s_{f}(q)\left(\sum_{a \leq H} \sum_{\substack{n \sim N \\
n \equiv a(q)}} f(n)-\frac{H}{q} \sum_{n \sim N} f(n)\right) \\
= & \sum_{a \leq H}\left(\sum_{n \sim N} f(n) f_{1}(n-a)-R_{1}\left(f_{1}\right) \sum_{n \sim N} f(n)\right) \\
= & \sum_{a \leq H} \sum_{N-a<x \leq 2 N-a} f(x+a) f_{1}(x)-R_{1}\left(f_{1}\right) R_{1}(f) N H \\
& +O_{\varepsilon}\left(N^{\varepsilon} Q H\right) \\
= & \sum_{x \sim N} f_{1}(x) \sum_{x<m \leq x+H} f(m)-R_{1}\left(f_{1}\right) R_{1}(f) N H \\
& +O_{\varepsilon}\left(N^{\varepsilon}(Q+H) H\right) \\
= & \sum_{x \sim N} f_{1}(x)\left(\sum_{x<m \leq x+H} f(m)-R_{1}(f) H\right) \\
& +O_{\varepsilon}\left(N^{\varepsilon}(Q+H) H\right),
\end{aligned}
$$

where we have applied (1..3) to both $f$ and $f_{1}$. Note that the $O$-term contribution is non-trivial because of hypotheses on $Q$ and $H$. In order to deal with the main term of the latter formula, after recalling that $f_{1}$ is essentially bounded, we apply the Cauchy inequality and the above assumption on $J_{f}(N, H)$ to get

$$
\begin{aligned}
\sum_{x \sim N} f_{1}(x)\left(\sum_{x<m \leq x+H} f(m)-R_{1}(f) H\right) & \ll_{\varepsilon} N^{1 / 2+\varepsilon} \sqrt{J_{f}(N, H)} \\
& \ll_{\varepsilon} N^{1+\varepsilon-\delta / 2} H,
\end{aligned}
$$


which in turn yields

$$
\sum_{q \leq Q}\left|T_{f}(q, N, H)\right| \ll N^{1-\delta / 3} H
$$

Theorem 1.9 is completely proved.

\section{A remarkable truncated divisor sum}

Let us recall that the truncated divisor sum defined in [Gol92] is

$$
\Lambda_{R}(n) \stackrel{\text { def }}{=} \sum_{\substack{d \mid n \\ d \leq R}} \mu(d) \log (R / d)=(\log R) \sum_{\substack{d \mid n \\ d \leq R}} \mu(d)-\sum_{\substack{d \mid n \\ d \leq R}} \mu(d) \log d,
$$

so that $\Lambda_{R}$ is plainly a linear combination (with relatively small coefficients) of two sieve functions, whose Eratosthenes transforms are respectively the restricted Möbius function, $\mu_{R} \stackrel{\text { def }}{=} \mu \cdot \mathbf{1}_{[1, R]}$, and $\mu_{R} \cdot \log$.

After recalling also the well-known relations (see [Da00])

$$
\sum_{d=1}^{\infty} \frac{\mu(d) \log d}{d}=-1 \quad \text { and } \quad \sum_{d \leq R} \frac{\mu(d)}{d}, \sum_{d>R} \frac{\mu(d) \log d}{d} \ll \exp (-c \sqrt{\log R}),
$$

(hereafter, $c>0$ is an unspecified constant), we see that

$$
\begin{aligned}
R_{1}\left(\Lambda_{R}\right) & =\sum_{d \leq R} \frac{\mu(d) \log (R / d)}{d} \\
& =(\log R) \sum_{d \leq R} \frac{\mu(d)}{d}-\sum_{d \leq R} \frac{\mu(d) \log d}{d}=1+O(\exp (-c \sqrt{\log R})) .
\end{aligned}
$$

Thus, the mean value formula (1..3) gives

$$
\sum_{n \sim N} \Lambda_{R}(n)=N+O(N \exp (-c \sqrt{\log R}))+O_{\varepsilon}\left(N^{\varepsilon} R\right)
$$

while, if $R \ll N$, a straightforward application of (1..1) yields

$$
\sum_{a \leq H} \sum_{\substack{n \sim N \\ n \equiv a(q)}} \Lambda_{R}(n)=\frac{N H}{q}+O_{\varepsilon}\left(N^{\varepsilon}\left(\frac{N}{q}+q+R\right)\right)+O(N \exp (-c \sqrt{\log R})) .
$$

In case the level $\lambda \stackrel{\text { def }}{=}(\log R) /(\log N)$ is positive, i.e. $0<\lambda_{0} \leq \lambda<1$ (for a fixed $\lambda_{0}$ ), we may replace $\log R$ by $\log N$ in the above formulæ, where now $c=c(\lambda)$. Assuming that this is the case, Corollary 1.7 provides the following first generation formula for the correlation of $\Lambda_{R}$ :

$$
\begin{aligned}
\sum_{a \leq H} \sum_{n \sim N} \Lambda_{R}(n) \Lambda_{R}(n-a)= & N H+O(N H \exp (-c \sqrt{\log N})) \\
& +O_{\varepsilon}\left(N^{\varepsilon}\left(N+R^{2}+R H\right)\right) .
\end{aligned}
$$

It is worthwhile to remark that by following the classical approach in the literature the remainder term for the single correlation is $\ll_{\varepsilon} N^{\varepsilon} R^{2}$, that trivially yields a remainder $\ll_{\varepsilon} N^{\varepsilon} R^{2} H$ in the first generation formula above, whereas by our method we save $H$. 


\section{Further comments}

The key of the present approach is that the correlation of a real sieve function $f=g_{Q} * \mathbf{1}$ can be written as

$$
\mathcal{C}_{f}(a)=\sum_{q \leq Q} g(q) \sum_{\substack{n \sim N \\ n \equiv a(q)}} f(n) .
$$

In the literature (see [IwaKow04], Ch.17), we find several studies of the distribution of an arithmetic function $f$ (not necessarily a sieve function) over primitive residue classes. Most results are focused on non-trivial bounds for the error term

$$
E_{f}(N ; q, a) \stackrel{\text { def }}{=} \sum_{\substack{n \sim N \\ n \equiv a(q)}} f(n)-M_{f}(N ; q, a)
$$

for all $(a, q)=1$, provided $q$ is not too large. Here, $M_{f}(N ; q, a)$ is the expected mean value term. Let us recall two major variants of the problem. The first one concerns the Bombieri-Vinogradov type mean

$$
\sum_{q \leq Q} \max _{(a, q)=1}\left|E_{f}(N ; q, a)\right|,
$$

for which we refer the reader to [Mot76]. The second classical variant is the Barban-DavenportHalberstam type quadratic mean

$$
\sum_{q \leq Q} \sum_{\substack{a \leq q \\(a, q)=1}} E_{f}(N ; q, a)^{2} .
$$

The latter has also a short interval version introduced by Hooley [Hoo99], that is

$$
\sum_{q \leq Q} \sum_{\substack{a \leq \rho q \\(a, q)=1}} E_{f}(N ; q, a)^{2}, \text { where } \rho \rightarrow 0 .
$$

In all such problems, the challenging issue is the level $\lambda \stackrel{\text { def }}{=}(\log Q) /(\log N)$ of distribution of $f$ in arithmetic progressions (see [FriIwa10], §9.8 and §22.1). For example, the celebrated BombieriVinogradov Theorem gives a non-trivial bound for

$$
\sum_{q \leq Q} \max _{(a, q)=1}\left|\sum_{\substack{n \sim N \\ n \equiv a(q)}} \Lambda(n)-\frac{N}{\varphi(q)}\right|, \quad \text { where } \varphi(q) \stackrel{\text { def }}{=}|\{a \leq q,(a, q)=1\}|,
$$

essentially with a level $\lambda=1 / 2$ (which seems to be a structural barrier at least for the distribution of primes). However, for many applications one can just deal with individual reduced class $a$ and take the sum over $q \leq Q,(q, a)=1$. Indeed, by assuming that $a \neq 0$, one can see that it is possible to break the level 1/2 for the Bombieri-Friedlander-Iwaniec type mean (see [FriIwa10], Theorem 22.1)

$$
\sum_{\substack{q \leq Q \\(a, q)=1}}\left|\sum_{\substack{n \sim N \\ n \equiv a(q)}} \Lambda(n)-\frac{N}{\varphi(q)}\right|
$$

Consistently with the present notation, the above formula for the correlation of a sieve function becomes

$$
\mathcal{C}_{f}(a)=\sum_{q \leq Q} g(q) M_{f}(N ; q, a)+\sum_{q \leq Q} g(q) E_{f}(N ; q, a),
$$

where, by recalling that $g(q) \ll_{\varepsilon} q^{\varepsilon}$, one has

$$
\sum_{q \leq Q} g(q) E_{f}(N ; q, a) \ll_{\varepsilon} Q^{\varepsilon} \sum_{q \leq Q}\left|E_{f}(N ; q, a)\right|
$$


Thus, here for each individual residue $a$ we deal with a sum over $q \leq Q$ without any further restriction. Then, it is not surprising that a straight asymptotic

$$
\mathcal{C}_{f}(a) \sim \sum_{q \leq Q} g(q) M_{f}(N ; q, a)
$$

has been proved for very few interesting instances of $f$, including the noteworthy case of the divisor function (see the third version of [CopLap14] on arXiv for a brief account on this matter). Better expectations for the first generation of correlation averages,

$$
\sum_{a \leq H} \mathfrak{e}_{f}(a)
$$

are given substance by Corollary 1.7 (and by the alternative approach of Lemma 12 in [CopLap14]). Furthermore, note that Theorem 1.9 concerns the average

$$
\sum_{q \leq Q}\left|\sum_{a \leq H} E_{f}(N ; q, a)\right|
$$

where, unlike the aforementioned means, the sums are taken over all the moduli $q \leq Q$ and over a short interval of residue classes $a$, when $f$ is a sieve function of range $Q \ll N^{1-\delta}$ and $H \ll N^{1-\delta}$. The bound for the weighted Selberg integral given in Corollary 1.7 and its application through Theorem 1.9 allow $Q \ll \sqrt{N H} N^{-\varepsilon}$, that is to say, the level might go beyond $1 / 2$ when we deal with not too short intervals, e.g., $H \gg N^{3 \varepsilon}$.

Acknowledgement. This research started while the first author was enjoying a fellowship entitled to Ing. Giorgio Schirillo by the Istituto Nazionale di Alta Matematica (Italy). The authors wish to thank the Referee for helpful comments and suggestions.

\section{References}

[Cop10a] G. Coppola, On the Correlations, Selberg integral and symmetry of sieve functions in short intervals, J. Comb. Number Theory, 2.2, Article 1, (2010), 91-105. MR-2907785

[Cop10b] G. Coppola, On the Correlations, Selberg integral and symmetry of sieve functions in short intervals, II, International Journal of Pure and Applied Mathematics, 58.3 (2010), 281-298. MR-2640394

[Cop14] G. Coppola, On some lower bounds of some symmetry integrals, Afr. Mat. 25, issue 1 (2014), 183-195. MR-3165958

[CopLap14] G. Coppola and M. Laporta, Generations of correlation averages, J. Numbers, Vol. 2014, Article ID 140840 (2014), $1-13$.

[CopLap15] G. Coppola and M. Laporta, On the Correlations, Selberg integral and symmetry of sieve functions in short intervals, III, Mosc. J. Comb. Number Theory, 6 (1), (2016), 3-24.

[CopLap16] G. Coppola and M. Laporta, Symmetry and short interval mean-squares, (2016), arXiv:1312.5701 (submitted).

[Da00] H. Davenport, Multiplicative Number Theory. 3rd edition, GTM 74, Springer, New York, 2000.

[DiaHal08] H.G. Diamond and H. Halberstam, A Higher-Dimensional Sieve Method, (With Procedures for Computing Sieve Functions by W.F. Galway). Cambridge Tracts in Mathematics, Vol. 177, Cambridge University Press, Cambridge, 2008.

[FriIwa10] J. Friedlander and H. Iwaniec, Opera de Cribro. AMS Colloquium Publications, 57, Providence, RI, 2010.

[Gol92] D.A. Goldston, On Bombieri and Davenport's theorem concerning small gaps between primes, Mathematika 39 (1992), 10-17.

[Hoo99] C. Hooley, On the Barban-Davenport-Halberstam Theorem. XI, Acta Arith. 91, no.1 (1999), 1-41.

[IwaKow04] H. Iwaniec and E. Kowalski, Analytic Number Theory. AMS Colloquium Publications, 53, Providence, RI, 2004

[Mot76] Y. Motohashi, An induction principle for the generalization of Bombieri's prime number theorem, Proc. Japan Acad. 52, no.6 (1976), 273-275.

[SchSpi94] W. Schwarz and J. Spilker, Arithmetical functions, (An introduction to elementary and analytic properties of arithmetic functions and to some of their almost-periodic properties). London Mathematical Society Lecture Note Series, 184, Cambridge University Press, Cambridge, 1994. 
[Win43] A. Wintner, Eratosthenian Averages. Waverly Press, Baltimore, MD, 1943.

\section{Giovanni Coppola}

Home address: Via Partenio 12,

83100, Avellino (AV), ITALY

e-page: www.giovannicoppola.name

e-mail: giocop@interfree.it

Maurizio Laporta

Università degli Studi di Napoli "Federico II",

Dipartimento di Matematica e Applicazioni "R.Caccioppoli",

Complesso di Monte S.Angelo, Via Cinthia - 80126, Napoli, ITALY

e-mail: mlaporta@unina.it 